\title{
ON CERTAIN INTEGRALS ASSOCIATED TO CR-FUNCTIONS
}

\author{
TELEMACHOS HATZIAFRATIS
}

\begin{abstract}
We construct explicit $\bar{\partial}$-primitives of Cauchy-Fantappiè type kernels on analytic subvarieties of domains in $\mathbf{C}^{n}$, outside the zero set of a holomorphic map defined on the variety. Then we use these primitives to derive, by means of a process of passing to a residue, integral formulas for CR-functions defined on subsets of the boundary of a variety. Certain geometric restrictions on the varieties are imposed. These primitives apply in the particular case of the Bochner-Martinelli kernel in domains in $\mathbf{C}^{n}$ and we use these primitives, in this case, to prove a criterion for holomorphic extendability of CR-functions defined on certain subsets of the boundary of a domain in $\mathbf{C}^{n}$.
\end{abstract}

\section{INTRODUCTION}

Let $D, G \subset \mathbf{C}^{n}$ be bounded domains with smooth boundary and $\bar{D} \subset G$. Let $h: G \rightarrow \mathbf{C}^{p}$ be a holomorphic map and set $Z(h)=\{\zeta \in G: h(\zeta)=0\}$. In [2] we constructed kernels $\alpha^{h}(\zeta, z)$ and $\beta^{h}(\zeta)$ so that if $f$ is a holomorphic function on $Z(h)$ and $z \in Z(h) \cap D$ then

$$
f(z)=\int_{\zeta \in Z(h) \cap \partial D} f(\zeta) \alpha^{h}(\zeta, z) \wedge \beta^{h}(\zeta) .
$$

The differential forms $\alpha^{h}$ and $\beta^{h}$ were explicitly defined in terms of $h$, a Hefer decomposition $\left\{h_{i j}\right\}$ of $h$, i.e., holomorphic functions $h_{i j}(\zeta, z)$ in $G \times G$ with

$$
h_{i}(\zeta)-h_{i}(z)=\sum_{j=1}^{n} h_{i j}(\zeta, z)\left(\zeta_{j}-z_{j}\right), \quad i=1, \ldots, p,(\zeta, z) \in G \times G,
$$

and in terms of a function $\gamma:(\partial D) \times D \rightarrow \mathbf{C}^{n}$ so that

$$
\sum_{j=1}^{n} \gamma_{j}(\zeta, z)\left(\zeta_{j}-z_{j}\right)=1 \quad \text { for }(\zeta, z) \in(\partial D) \times D .
$$

Certain geometric restrictions (transversality conditions) were imposed which guarantee that $Z(h) \cap \partial D$ is a smooth manifold.

In this paper we pursue further these constructions by proving an integral formula satisfied by CR-functions which are defined on certain parts of the boundary of a variety. First we construct another kernel, $\eta_{g}^{h}(\zeta, z)$, which is a

Received by the editors February 15, 1988.

1980 Mathematics Subject Classification (1985 Revision). Primary 32A25, 32A27, 32D15. 
$\bar{\partial}_{\zeta}$-primitive of $\alpha^{h}(\zeta, z)$ on $\operatorname{Dom}(\gamma(\cdot, z)) \cap Z(h)$ off the zero variety $Z(g)=$ $\{\zeta \in G: g(\zeta)=0\}$ of $g$, where $g: G \rightarrow \mathbf{C}^{q}$ is another holomorphic map with $p+q \leq n-1$ and $z \in Z(h, g) \cap D$ is fixed, i.e.,

$$
\bar{\partial}_{\zeta} \eta_{g}^{h}(\zeta, z)=\alpha^{h}(\zeta, z) \quad \text { for } \zeta \in \operatorname{Dom}(\gamma(\cdot, z)) \cap(Z(h)-Z(h, g)) .
$$

The kernel $\eta_{g}^{h}(\zeta, z)$ is explicitly defined in terms of $\gamma$, of $g$ and of Hefer decompositions of $h$ and $g$ and it is singular along $Z(h, g)$; see $\S 1$ for the precise description of $\eta_{g}^{h}$. This construction is interesting even in the case $p=0$ in which case $Z(h)=G$ and $\alpha^{h}(\zeta, z) \wedge \beta^{h}(\zeta)$ is simply the Cauchy-Fantappiè kernel, $\omega^{\prime}(\gamma)(\zeta, z) \wedge \omega(\zeta)$, associated to $\gamma$ (see $\S 3$ for notation). The case $p=$ $0, q=1$ was carried out earlier in $\left[7,5\right.$ and 3] with $\gamma_{j}=|\zeta-z|^{-2}\left(\bar{\zeta}_{j}-\bar{z}_{j}\right)$. In these papers the $\bar{\partial}_{\zeta}$-primitive (which coincides with ours in this case) was used to prove certain theorems about holomorphic extendability of CR-functions.

In $\S 2$ we consider the subvarieties $Z(h) \cap D$ and $Z(g) \cap D$ of $D$ (with $h$ and $g$ as before), open subsets $\Gamma, \widetilde{\Gamma} \subset Z(h) \cap \partial D$ (open in $Z(h) \cap \partial D$ ) with $\bar{\Gamma} \subset \widetilde{\Gamma}$ and $Z(h, g) \cap \partial D \subset \Gamma$ and a CR-function $f$ on $\widetilde{\Gamma}$ and we prove that

$$
\begin{gathered}
\int_{\zeta \in \Gamma} f(\zeta) \alpha^{h}(\zeta, z) \wedge \beta^{h}(\zeta)-\int_{\zeta \in \partial \Gamma} f(\zeta) \eta_{g}^{h}(\zeta, z) \wedge \beta^{h}(\zeta) \\
=\int_{\zeta \in Z(h, g) \cap \partial D} f(\zeta) \alpha^{(h, g)}(\zeta, z) \wedge \beta^{(h, g)}(\zeta)
\end{gathered}
$$

for $z \in Z(h, g) \cap D$.

Let us point out that the integrand of the integral $\int_{\partial \Gamma}$ in (3) is singular along a certain part of $\Gamma$, namely $Z(h, g) \cap \partial D$, which accounts for the term $\int_{Z(h, g) \cap \partial D}$ in the right-hand side of $(3)$. Certain transversality assumptions are made which make Stokes' theorem type analysis on various manifolds possible. The derivation of (3) is based on Stokes' theorem, used to change the contour $\partial \Gamma$ in the integral $\int_{\partial \Gamma}$ into another contour, close to the singularities $Z(h, g) \cap$ $\partial D$ of $\eta_{g}^{h}(\zeta, z)$, together with a process of passing to a residue; a particular case of this process was carried out in [6].

The integral formula (3) is a generalization of (1) since, in the particular case $f$ extends to a holomorphic function on $G, p=0$ (i.e., $Z(h)=G$ ) and $\Gamma=Z(h) \cap \partial D=\partial D$ (in which case $\partial \Gamma=\dot{\varnothing}$ ), the integral formula (3) is reduced to

$$
\int_{\zeta \in \partial D} f(\zeta) \omega^{\prime}(\gamma)(\zeta, z) \wedge \omega(\zeta)=\int_{\zeta \in Z(g) \cap \partial D} f(\zeta) \alpha^{g}(\zeta, z) \wedge \beta^{g}(\zeta)
$$

and the integral in the left-hand side of (4) is equal to $f(z)$ by the CauchyFantappiè formula. The interest in (3) lies, in part, in that this integral formula holds regardless of the existence or not of an extension of $f$, from $\widetilde{\Gamma}$ to any larger set, as a CR-or holomorphic function. Formula (3) is proved in $\S 2$ where we derive some other integral formulas which follow from it. 
Finally, in $\S 3$, we specialize in domains in $\mathbf{C}^{n}$ and we use the $\bar{\partial}$-primitives of the Bochner-Martinelli kernel that we constructed, to prove a criterion for holomorphic extendability of CR-functions defined on certain parts of the boundary of a domain in $\mathbf{C}^{n}$. This criterion is stated in terms of certain integrals of the primitives (involving the CR-function too) taken on cycles lying in the part of the boundary of the domain where the CR-function is defined. In this paragraph we follow ideas from [3, 4 and 7$]$.

Acknowledgments. I was able to carry out some parts of this paper after discussions that I had with Professors Lee Stout and Guido Lupacciolu, whom I would like to thank.

\section{A $\bar{\partial}$-PRIMITIVE}

In this section we will construct explicit $\bar{\partial}$-primitives for some CauchyFantappiè type kernels on analytic varieties. We start by describing the setting and establishing notation. Let $D$ and $G$ be bounded domains in $\mathbf{C}^{n}$ with smooth boundary and $\bar{D} \subset G$. Let $\gamma:(\partial D) \times D \rightarrow \mathbf{C}^{n}$ be a smooth function so that

$$
\sum_{j=1}^{n} \gamma_{j}(\zeta, z)\left(\zeta_{j}-z_{j}\right)=1 \text { for }(\zeta, z) \in(\partial D) \times D .
$$

For a holomorphic map $h: G \rightarrow \mathbf{C}^{p}$ and a Hefer decomposition $\left\{h_{i j}\right\}$ of it, i.e., holomorphic functions $h_{i j}(\zeta, z)$ in $G \times G$ with

$$
(* *) \sum_{j=1}^{n} h_{i j}(\zeta, z)\left(\zeta_{j}-z_{j}\right)=h_{i}(\zeta)-h_{i}(z), \quad i=1, \ldots, p,(\zeta, z) \in G \times G,
$$

we associate the differential form $\alpha^{h}(\zeta, z)$ in the following way:

$$
\alpha^{h}(\zeta, z)=c(p) \operatorname{det}[h_{1 j}, \ldots, h_{p j}, \gamma_{j}, \overbrace{\overbrace{\zeta} \gamma_{j}}^{n-p-1}]_{1 \leq j \leq n}
$$

where $c(p)=(-1)^{p n} \cdot(-1)^{\frac{n(n-1)}{2}} \cdot(2 \pi i)^{p-n}$; here we use determinants with entries differential forms; for properties of such determinants see [1, p. 8]. In the above determinant which defines $\alpha^{h}, j$ runs from $j=1$ to $j=n$ forming the $n$-rows of it and the column $\left[\bar{\partial}_{\zeta} \gamma_{j}\right]_{1 \leq j \leq n}$ is repeated $(n-p-1)$-times (as indicated); $\alpha^{h}$ is a $(0, n-p-1)$-form in $\zeta$. Of course, the differential form $\alpha^{h}(\zeta, z)$ depends on $\gamma$ and on the chosen Hefer decomposition $\left\{h_{i j}\right\}$ of $h$ which we consider fixed. Let us point out that for most of what follows, $z$ will be a fixed point inside $D$ and $\zeta$ will belong to $\partial D$. Also, if we fix $z$ in $D$ then there is a smooth extension of $\gamma(\cdot, z)$ (in $\zeta$ ) in a neighborhood of $\partial D$, satisfying (*) and we will frequently make use of such an extension. 
We will also use multi-index notation which we describe next. For a $p$-multiindex $J=\left\{1 \leq j_{1}<\cdots<j_{p} \leq n\right\}$ we write

$$
D_{J}(h)=\frac{\partial\left(h_{1}, \ldots, h_{p}\right)}{\partial\left(\zeta_{j_{1}}, \ldots, \zeta_{j_{p}}\right)} \quad \text { and } \quad(-1)^{J}=(-1)^{j_{1}+\cdots+j_{p}}
$$

For an $r$-multi-index $I=\left\{1 \leq i_{1}<\cdots<i_{r} \leq n\right\}$ we will write $d \zeta_{[I]}$ for the following differential form

$$
d \zeta_{[I]}=d \zeta_{i_{1}} \wedge \cdots \wedge d \zeta_{i_{r}}
$$

Let $N$ denote the particular $n$-multi-index

$$
N=\{1 \leq 1<\cdots<n \leq n\} .
$$

In particular

Define

$$
d \zeta_{[N-J]}=\bigwedge_{\substack{k \neq j_{1}, \ldots, j_{p} \\ k=1}}^{n} d \zeta_{k}=\bigwedge_{k \in N-J} d \zeta_{k}
$$

$$
\beta^{h}(\zeta)=|\nabla h(\zeta)|^{-2} \sum_{J \sim p}(-1)^{J} \overline{D_{J}(h)(\zeta)} d \zeta_{[N-J]}
$$

where $|\nabla h|=\left(\sum_{J \sim p}\left|D_{J}(h)\right|^{2}\right)^{1 / 2}$ and $\sum_{J \sim p}$ indicates that the summation is extended over all ordered $p$-multi-indices $J=\left\{j_{1}<\cdots<j_{p}\right\} \subset\{1, \ldots, n\}$. Of course $\beta^{h}(\zeta)$ is defined for $\zeta$ for which $|\nabla h(\zeta)| \neq 0$.

Now let $g: G \rightarrow C^{q}$ be another holomorphic map with $p+q \leq n-1$ and let $\left\{g_{k j}\right\}$ be a Hefer decomposition of $g$, i.e., $g_{k j}$ are holomorphic functions on $G \times G$ with

$$
(* * *) \sum_{j=1}^{n} g_{k j}(\zeta, z)\left(\zeta_{j}-z_{j}\right)=g_{k}(\zeta)-g_{k}(z), \quad k=1, \ldots, q,(\zeta, z) \in G \times G
$$

Then $(h, g)=\left(h_{1}, \ldots, h_{p}, g_{1}, \ldots, g_{q}\right): G \rightarrow \mathbf{C}^{p+q}$ is a holomorphic map too and therefore we can associate to it, to the chosen Hefer decompositions $\left\{h_{i j}, g_{k j}\right\}$ of it and to $\gamma$, the differential forms $\alpha^{(h, g)}(\zeta, z)$ and $\beta^{(h, g)}(\zeta)$; let us point out that the constant in front of $\alpha^{(h, g)}$ is $c(p+q) ; \alpha^{(h, g)}$ is a $(0, n-p-q-1)$-form (in $\zeta)$ and $\beta^{(h, g)}$ is a $(n-p-q, 0)$-form. Similarly we can define $\alpha^{g}(\zeta, z)$ and $\beta^{g}(\zeta)$.

Next we describe the geometric assumptions that we make in this section. Let

$$
Z(h)=\{\zeta \in G: h(\zeta)=0\} .
$$

We assume that $|\nabla h(\zeta)| \neq 0$ for $\zeta \in Z(h) \cap \partial D$ so that $Z(h)$ is smooth near $\partial D$ and that $Z(h)$ meets $\partial D$ transversally so that $Z(h) \cap \partial D$ is a smooth $(2 n-2 p-1)$-real-dimensional manifold; also $Z(h) \cap D$ has finitely many singular points. In this paragraph we do not make any assumption of independence of the $g_{1}, \ldots, g_{q}$; for example it may be that $g_{1}=\cdots=g_{q}$. 
In the above setting we will construct an explicit $\bar{\partial}_{\zeta}$-primitive for each of the differential forms $\alpha^{h}(\zeta, z)$ and $\alpha^{h}(\zeta, z) \wedge \beta^{h}(\zeta)$ restricted, in $\zeta$, to the variety $Z(h)$ outside of $Z(h, g) ; z$ will be a fixed point in $Z(h, g) \cap D$. For this purpose let us set

$$
s_{j}^{g}(\zeta, z)=\left(\sum_{k=1}^{q}\left|g_{k}(\zeta)\right|^{2}\right)^{-1} \sum_{k=1}^{q} g_{k j}(\zeta, z) \overline{g_{k}(\zeta)}, \quad j=1, \ldots, n,
$$

where $z$ is a fixed point in $Z(h, g) \cap D$ and $\zeta \in Z(h)-Z(h, g)$; notice that $(* * * *) \quad \sum_{j=1}^{n} s_{j}^{g}(\zeta, z)\left(\zeta_{j}-z_{j}\right)=1 \quad$ (this follows from $\left.(* * *)\right)$.

Define

$$
\eta_{g}^{h}(\zeta, z)=-c(p) \sum_{l=0}^{n-p-2} \operatorname{det}[h_{1 j}, \ldots, h_{p j}, \gamma_{j}, s_{j}^{g}, \overbrace{\bar{\partial}_{\zeta} \gamma_{j}}^{l}, \overbrace{\bar{\partial}_{\zeta} s_{j}^{g}}^{n-p-l-2}]_{1 \leq j \leq n} .
$$

Then $\eta_{g}^{h}(\zeta, z)$ is a differential form in $\zeta$ of type $(0, n-p-2)$. With this notation we will prove the following theorem.

Theorem 1.1. For a fixed $z \in Z(h, g) \cap D$ and with differential forms restricted, in $\zeta$, to $(Z(h)-Z(h, g)) \cap \operatorname{Dom}(\gamma(\cdot, z))$ we have

$$
\bar{\partial}_{\zeta} \eta_{g}^{h}(\zeta, z)=\alpha^{h}(\zeta, z)
$$

and

$$
d_{\zeta}\left[\eta_{g}^{h}(\zeta, z) \wedge \beta^{h}(\zeta)\right]=\bar{\partial}_{\zeta}\left[\eta_{g}^{h}(\zeta, z) \wedge \beta^{h}(\zeta)\right]=\alpha^{h}(\zeta, z) \wedge \beta^{h}(\zeta)
$$

Remarks. (a) Let us point out that the differential form $\eta_{g}^{h}$ depends on the chosen Hefer decompositions of $h$ and $g$ as well as on $\gamma$ which are considered fixed.

(b) Theorem 1.1 applies in the case $p=0$ too; in this case the kernel $\alpha^{h}(\zeta, z) \wedge \beta^{h}(\zeta)$ is simply the Cauchy-Fantappiè kernel of $D$ associated to $\gamma$ and Theorem 1.1 gives an explicit $\bar{\partial}_{\zeta}$-primitive of it in $(\partial D)-Z(g)$; we will come back to this point in $\S 3$.

Proof of Theorem 1.1. We may assume that $z=0 \quad(\in Z(h, g) \cap D)$; we may also assume, without loss of generality (because of the symmetry expressed by determinants), that $\zeta_{1} \neq 0$. Then

(1)

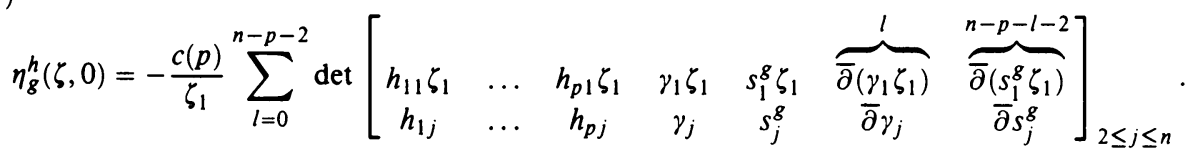

Throughout this proof, $\gamma_{j}=\gamma_{j}(\zeta, 0), h_{i j}=h_{i j}(\zeta, 0)$ and $s_{j}^{g}=s_{j}^{g}(\zeta, 0)$; also $\bar{\partial}=\bar{\partial}_{\zeta}$. In the determinants in (1) $j$ runs from $j=2$ to $j=n$ forming the 
2 nd up to the $n$ th-row of it, as indicated. Now let us multiply the $j$ th-row of each determinant in (1) by $\zeta_{j}$ and add it to its first row, for $j=2,3, \ldots, n$; then (1) becomes (in view of $(*),(* *)$ and $(* * * *)$ and the fact that $\zeta \in Z(h)$ and $0 \in Z(h, g))$ :

(2)

$$
\eta_{g}^{h}(\zeta, 0)=-\frac{c(p)}{\zeta_{1}} \sum_{l=0}^{n-p-2} \operatorname{det}\left[\begin{array}{ccccccc}
0 & \ldots & 0 & 1 & 1 & \overbrace{0}^{l} & \overbrace{0}^{n-p-l-2} \\
h_{1 j} & \ldots & h_{p j} & \gamma_{j} & s_{j}^{g} & \bar{\partial} \gamma_{j} & \bar{\partial} s_{j}^{g}
\end{array}\right]_{2 \leq j \leq n} .
$$

Next applying $\bar{\partial}_{\zeta}$ in both sides of (2) we obtain

(3)

$$
\begin{aligned}
& \bar{\partial}_{\zeta} \eta_{g}^{h}(\zeta, 0)=-\frac{c(p)}{\zeta_{1}} \sum_{l=0}^{n-p-2}\left(\operatorname{det}\left[\begin{array}{ccccccc}
0^{\prime} & \ldots & 0 & 0 & 1 & \overbrace{0}^{l} & \overbrace{\frac{0}{\partial} s_{j}^{g}}^{n-p-l-2} \\
h_{1 j} & \ldots & h_{p j} & \bar{\partial} \gamma_{j} & s_{j}^{g} & \frac{\bar{\partial} \gamma_{j}}{n_{j}}
\end{array}\right]\right. \\
& \left.+\operatorname{det}\left[\begin{array}{ccccccc}
0 & \ldots & 0 & 1 & 0 & \overbrace{0}^{l} & \overbrace{\overline{0}}^{n-p-l-2} \\
h_{1 j} & \ldots & h_{p j} & \gamma_{j} & \bar{\partial} s_{j}^{g} & \frac{\bar{\partial} \gamma_{j}}{\partial s_{j}^{g}}
\end{array}\right]\right) \\
& =(-1)^{p+1} \frac{c(p)}{\zeta_{1}} \sum_{l=0}^{n-p-2}\left(\operatorname{det}[h_{1 j}, \ldots, h_{p j}, \bar{\partial} s_{j}^{g}, \overbrace{\bar{\partial} \gamma_{j}}^{l}, \overbrace{\bar{\partial} s_{j}^{g}}^{n-p-l-2}]_{2 \leq j \leq n}\right. \\
& \left.-\operatorname{det}[h_{1 j}, \ldots, h_{p j}, \bar{\partial} \gamma_{j}, \overbrace{\bar{\partial} \gamma_{j}}^{l}, \overbrace{\bar{\partial} s_{j}^{g}}^{n-p-l-2}]_{2 \leq j \leq n}\right) .
\end{aligned}
$$

Setting

$$
A_{l}=: \operatorname{det}[h_{1 j}, \ldots, h_{p j}, \overbrace{\bar{\partial} \gamma_{j}}^{l}, \overbrace{\bar{\partial} s_{j}^{g}}^{n-p-l-1}]_{2 \leq j \leq n}
$$

for $0 \leq l \leq n-p-1$, (3) can be written

$$
\begin{aligned}
\bar{\partial}_{\zeta} \eta_{g}^{h}(\zeta, 0) & =(-1)^{p+1} \frac{c(p)}{\zeta_{1}} \sum_{l=0}^{n-p-2}\left(A_{l}-A_{l+1}\right) \\
& =(-1)^{p+1} \frac{c(p)}{\zeta_{1}}\left(A_{0}-A_{n-p-1}\right) \\
& =(-1)^{p} \frac{c(p)}{\zeta_{1}}\left(A_{n-p-1}-A_{0}\right) .
\end{aligned}
$$


On the other hand,

$$
\begin{aligned}
& \alpha^{h}(\zeta, 0)=c(p) \operatorname{det}[h_{1 j}, \ldots, h_{p j}, \gamma_{j}, \overbrace{\bar{\partial} \gamma_{j}}^{n-p-1}]_{1 \leq j \leq n} \\
& =\frac{c(p)}{\zeta_{1}} \operatorname{det}\left[\begin{array}{ccccc}
h_{11} \zeta_{1} & \ldots & h_{p 1} \zeta_{1} & \gamma_{1} \zeta_{1} & \overbrace{\bar{\partial}\left(\gamma_{1} \zeta_{1}\right)}^{n-p-1} \\
h_{1 j} & \ldots & h_{p j} & \gamma_{j} & \bar{\partial} \gamma_{j}
\end{array}\right]_{2 \leq j \leq n} \\
& =\frac{c(p)}{\zeta_{1}} \operatorname{det}\left[\begin{array}{ccccc}
0 & \ldots & 0 & 1 & \overbrace{0}^{n-p-1} \\
h_{1 j} & \ldots & h_{p j} & \gamma_{j} & \bar{\partial} \gamma_{j}
\end{array}\right]_{2 \leq j \leq n} \\
& =(-1)^{p} \frac{c(p)}{\zeta_{1}} \operatorname{det}[h_{1 j}, \ldots, h_{p j}, \overbrace{\bar{\partial} \gamma_{j}}^{n-p-1}]_{2 \leq j \leq n} \\
& =(-1)^{p} \frac{c(p)}{\zeta_{1}} A_{n-p-1} .
\end{aligned}
$$

Similarly we prove (in the same way we proved (5)) that

$$
\begin{aligned}
\frac{(-1)^{p} A_{0}}{\zeta_{1}} & =\frac{(-1)^{p}}{\zeta_{1}} \operatorname{det}[h_{1 j}, \ldots, h_{p j}, \overbrace{\bar{\partial} s_{j}^{g}}^{n-p-1}]_{2 \leq j \leq n} \\
& =\operatorname{det}[h_{1 j}, \ldots, h_{p j}, s_{j}^{g}, \overbrace{\bar{\partial} s_{j}^{g}}^{n-p-1}]_{1 \leq j \leq n} \\
& =\left(\sum_{k=1}^{q}\left|g_{k}(\zeta)\right|^{2}\right)^{p-n} \operatorname{det}[h_{1 j}, \ldots, h_{p j}, \tilde{s}_{j}, \overbrace{\bar{\partial} \tilde{s}_{j}}^{n-p-1}]_{1 \leq j \leq n}
\end{aligned}
$$

where we have set: $\tilde{s}_{j}=\sum_{k=1}^{q} g_{k j} \bar{g}_{k}$ (notice that $\sum_{j=1}^{n} \tilde{s}_{j} \cdot \zeta_{j}=\sum_{k=1}^{q}\left|g_{k}\right|^{2}$ ). The last equation in (6) follows from properties of determinants (see [2, Lemma 4, p. 80]).

Observing that

$$
\bar{\partial} \tilde{s}_{j}=\sum_{k=1}^{q} g_{k j} \overline{\partial g_{k}}
$$

we claim that

$$
\operatorname{det}[h_{1 j}, \ldots, h_{p j}, \tilde{s}_{j}, \overbrace{\bar{\partial} \tilde{s}_{j}}^{n-p-1}]_{1 \leq j \leq n}=0 .
$$

To prove (8) we distinguish two cases: 
1 st case: $p+q \leq n-2$, i.e., $q \leq n-p-2$; in this case we have

$$
\bar{\partial} \tilde{s}_{j_{1}} \wedge \cdots \wedge \bar{\partial} \tilde{s}_{j_{n-p-1}}=0
$$

for $1 \leq j_{1}<\cdots<j_{n-p-1} \leq n$; this follows from (7); but (9) implies (8) in this case.

2nd case: $p+q=n-1$, i.e., $q=n-p-1$; in this case it follows again from (7) and multilinearity of determinants that

(10)

$$
\begin{aligned}
\operatorname{det} & {[h_{1 j}, \ldots, h_{p j}, \tilde{s}_{j}, \overbrace{\bar{\partial} \tilde{s}_{j}}^{n-p-1}]_{1 \leq j \leq n} } \\
& =q ! \operatorname{det}\left[h_{1 j}, \ldots, h_{p j}, \sum_{k=1}^{q} g_{k j} \bar{g}_{k}, g_{1 j}, \ldots, g_{q j}\right] \overline{\partial g_{1}} \wedge \cdots \wedge \overline{\partial g_{q}}=0 ;
\end{aligned}
$$

the last equation in (10) follows from the fact that the $(p+1)$ th-column in the second determinant is a linear combination of its last $q$ columns; this proves (8) in this case too.

Now (8), (6), (5) and (4) prove part (i) of Theorem 1.1; part (ii) of it follows from part (i) together with [2, Corollary 1, p. 76]; this completes the proof of Theorem 1.1.

\section{AN INTEGRAL FORMULA}

In this paragraph we will prove an integral formula involving CR-functions which are defined on subsets of the boundary of a variety. We will use the notation of $\S 1$; let $D, G, \gamma, h, g, h_{i j}, g_{k j}$ be as in $\S 1$. The geometric assumptions imposed in this paragraph are the following:

First we assume that $|\nabla h| \neq 0$ on $Z(h) \cap \partial D$ and that $Z(h)$ meets $\partial D$ transversally (as in $\S 1$ ); furthermore we assume that $|\nabla(h, g)| \neq 0$ on $Z(h, g) \cap$ $\partial D$ and that $Z(h, g)$ meets $Z(h) \cap \partial D$ transversally, so that $Z(h, g) \cap \partial D$ is a smooth manifold of real dimension $2 n-2 p-2 q-1$ and that $Z(h, g) \cap D$ has finitely many singular points; let us point out that, since we assume $p+q \leq n-1$, $\operatorname{dim}_{\mathrm{C}} Z(h, g) \geq 1$. Let us consider open sets $\Gamma, \widetilde{\Gamma} \subset Z(h) \cap \partial D$ (open in $Z(h) \cap \partial D)$ with $\bar{\Gamma} \subset \widetilde{\Gamma}$ and such that $Z(h, g) \cap \partial D \subset \Gamma ; \partial \Gamma$ is assumed to be smooth too. Having oriented $\mathbf{C}^{n}$ in the usual way, i.e., the volume form $\left(\frac{i}{2}\right)^{n} d \zeta_{1} \wedge d \bar{\zeta}_{1} \wedge \cdots \wedge d \zeta_{n} \wedge d \bar{\zeta}_{n}$ is positive, let $Z(h) \cap D$ have its natural orientation as a complex manifold (close to $Z(h) \cap \partial D$ ) and let $Z(h) \cap \partial D$ have the induced orientation. In this setting we will prove the following theorem. 
Theorem 2.1. For a smooth $C R$-function $f$ on $\widetilde{\Gamma}$ and $a z \in Z(h, g) \cap D$, the following integral formula holds:

$$
\begin{gathered}
\int_{\zeta \in \Gamma} f(\zeta) \alpha^{h}(\zeta, z) \wedge \beta^{h}(\zeta)-\int_{\zeta \in \partial \Gamma} f(\zeta) \eta_{g}^{h}(\zeta, z) \wedge \beta^{h}(\zeta) \\
=\int_{\zeta \in Z(h, g) \cap \partial D} f(\zeta) \alpha^{(h, g)}(\zeta, z) \wedge \beta^{(h, g)}(\zeta) .
\end{gathered}
$$

For the proof of this theorem we need the following lemmas; the first lemma gives an appropriate factorization of the differential form $\beta^{h}$.

Lemma 1. With differential forms restricted to the variety $Z(h)$, locally at a nonsingular point of it, we have

$$
\Omega(g) \wedge \beta^{(h, g)}=(-1)^{\frac{q(q+1)}{2}}(-1)^{p \cdot q} \beta^{h}
$$

where $\Omega(g)=\partial g_{1} \wedge \cdots \wedge \partial g_{q}$.

Lemma 2. If the differential form $\theta(\zeta)$ is given by the formula

$$
\theta(\zeta)=\sum_{J \sim p} C_{J}(\zeta) d \zeta_{[N-J]}
$$

for some functions $C_{J}(\zeta)$, then $\theta(\zeta)$, restricted to $Z(h)$ locally at a point where

$$
B(\zeta)=\frac{\partial\left(h_{1}, \ldots, h_{p}\right)}{\partial\left(\zeta_{n-p+1}, \ldots, \zeta_{n}\right)}(\zeta) \neq 0
$$

can be written in the following way:

$$
\theta(\zeta)=c^{*}(B(\zeta))^{-1}\left(\sum_{J \sim p}(-1)^{J} C_{J}(\zeta) D_{J}(h)(\zeta)\right) d \zeta_{1} \wedge \cdots \wedge d \zeta_{n-p}
$$

where

$$
c^{*}=(-1)^{p(n-p)} \cdot(-1)^{\frac{p(p+1)}{2}} .
$$

In particular,

$$
\beta^{h}(\zeta)=c^{*}(B(\zeta))^{-1} d \zeta_{1} \wedge \cdots \wedge d \zeta_{n-p}
$$

A proof of Lemma 2 is given in [2, p. 75]. We will use Lemma 2 to prove Lemma 1.

Proof of Lemma 1. We have

$$
\Omega(g)=\sum_{K \sim q} D_{K}(g) d \zeta_{[K]}
$$

Also, by definition of $\beta^{(h, g)}$,

$$
\beta^{(h, g)}=|\nabla(h, g)|^{-2} \sum_{\substack{J \sim p, K \sim q \\ J<K}}(-1)^{J \cup K} \overline{D_{J \cup K}(h, g)} d \zeta_{[N-(J \cup K)]}
$$

$(J<K$ simply means that $\max J<\min K)$. 
It follows from (1) and (2) (after a computation) that (3)

$\Omega(g) \wedge \beta^{(h, g)}=|\nabla(h, g)|^{-2} \sum_{J \sim p}(-1)^{J}\left[\sum_{K \sim q}(-1)^{K * J} D_{K}(g) \overline{D_{J \cup K}(h, g)}\right] d \zeta_{[N-J]}$

where the multi-index $K * J$ is defined in the following way:

let $J=\left\{j_{1}<\cdots<j_{p}\right\}$ and $K=\left\{i_{1}<\cdots<i_{q}\right\}$; then $J \cup K=\left\{\mu_{1}<\cdots<\mu_{p+q}\right\}$ (i.e., we have arranged the set $\left\{j_{1}, \ldots, j_{p}, i_{1}, \ldots, i_{q}\right\}$ in an increasing $(p+q)$-multi-index $)$. If the $i_{1}, \ldots, i_{q}$ are found in the $\lambda_{1}, \ldots, \lambda_{q}$ positions among the $\mu_{1}<\cdots<\mu_{p+q}\left(1 \leq \lambda_{1}<\cdots<\lambda_{q} \leq p+q\right)$ then

$$
K * J=\left\{\lambda_{1}<\cdots<\lambda_{q}\right\} \subset\{1,2, \ldots, p+q\} .
$$

(In (3) the multi-indices $J$ and $K$ are not related, i.e., in (3), we do not assume $J<K$ as we do in (2).)

Applying Lemma 2 with $\theta$ being the right-hand side of (3) we obtain

$$
\begin{aligned}
\Omega(g) & \wedge \beta^{(h, g)}=c^{*} B^{-1}|\nabla(h, g)|^{-2} \\
& \cdot \sum_{J \sim p}\left(\sum_{K \sim q}(-1)^{K * J} D_{K}(g) D_{J}(h) \overline{D_{J \cup K}(h, g)}\right) d \zeta_{1} \wedge \cdots \wedge d \zeta_{n-p} .
\end{aligned}
$$

It follows from the rule of expanding determinants by minors (applied to $D_{J \cup K}(g, h)$, i.e., expanded by the $q \times q$-minors of its first $q$-columns) and the definition of $|\nabla(h, g)|$, that

$$
\begin{aligned}
\sum_{J \sim p} & \left(\sum_{K \sim q}(-1)^{K * J} D_{K}(g) D_{J}(h) \overline{D_{J \cup K}(h, g)}\right) \\
& =(-1)^{\frac{q(q+1)}{2}} \cdot(-1)^{p q} \sum_{S \sim(p+q)}\left|D_{S}(h, g)\right|^{2} \\
& =(-1)^{\frac{q(q+1)}{2}}(-1)^{p q}|\nabla(h, g)|^{2} .
\end{aligned}
$$

From (4) and (5) we obtain

$$
\begin{aligned}
\Omega(g) \wedge \beta^{(h, g)} & =(-1)^{p q}(-1)^{\frac{q(q+1)}{2}} c^{*} B^{-1} d \zeta_{1} \wedge \cdots \wedge d \zeta_{n-p} \\
& =(-1)^{p q}(-1)^{\frac{q(q+1)}{2}} \beta^{h} .
\end{aligned}
$$

The last equation in (6) is the particular case mentioned in Lemma 2. This completes the proof of Lemma 1.

Now we start the proof of Theorem 2.1.

Proof of Theorem 2.1. For a small $\varepsilon>0$ let us consider the domain

$$
\Gamma^{\varepsilon}=\left\{\zeta \in \Gamma:\left|g_{1}(\zeta)\right|^{2}+\cdots+\left|g_{q}(\zeta)\right|^{2}>\varepsilon^{2}\right\}
$$


Then $\partial \Gamma^{\ell}=\partial \Gamma \cup T^{\ell}$ where

$$
T^{\varepsilon}=\left\{\zeta \in \Gamma:\left|g_{1}(\zeta)\right|^{2}+\cdots+\left|g_{q}(\zeta)\right|^{2}=\varepsilon^{2}\right\} .
$$

It follows from Sard's theorem that for a.e. $\varepsilon>0, T^{\ell}$ is a smooth manifold.

Applying Stokes' theorem to the differential form $d_{\zeta}\left[f(\zeta) \eta_{g}^{h}(\zeta, z) \wedge \beta^{h}(\zeta)\right]$ and the manifold $\Gamma^{\ell}$ we obtain

$$
\int_{\zeta \in \partial \Gamma^{e}} f(\zeta) \eta_{g}^{h}(\zeta, z) \wedge \beta^{h}(\zeta)=\int_{\zeta \in \Gamma^{e}} d_{\zeta}\left[f(\zeta) \eta_{g}^{h}(\zeta, z) \wedge \beta^{h}(\zeta)\right] .
$$

It follows from Theorem 1.1 and the fact that $f$ is a CR-function on $\widetilde{\Gamma}$, that

$$
\int_{\zeta \in \Gamma^{e}} d_{\zeta}\left[f(\zeta) \eta_{g}^{h}(\zeta, z) \wedge \beta^{h}(\zeta)\right]=\int_{\zeta \in \Gamma^{e}} f(\zeta) \alpha^{h}(\zeta, z) \wedge \beta^{h}(\zeta)
$$

But

$$
\int_{\partial \Gamma^{e}} f \eta_{g}^{h}(\cdot, z) \wedge \beta^{h}=\int_{\partial \Gamma} f \eta_{g}^{h}(\cdot, z) \wedge \beta^{h}+\int_{T^{e}} f \eta_{g}^{h}(\cdot, z) \wedge \beta^{h}
$$

It follows from (1), (2) and (3) that

$$
\int_{\Gamma^{e}} f \alpha^{h}(\cdot, z) \wedge \beta^{h}-\int_{\partial \Gamma} f \eta_{g}^{h}(\cdot, z) \wedge \beta^{h}=\int_{T^{\varepsilon}} f \eta_{g}^{h}(\cdot, z) \wedge \beta^{h} .
$$

Letting $\varepsilon \rightarrow 0$ through points so that $T^{\varepsilon}$ is smooth, we obtain from (4),

$$
\int_{\Gamma} f \alpha^{h}(\cdot, z) \wedge \beta^{h}-\int_{\partial \Gamma} f \eta_{g}^{h}(\cdot, z) \wedge \beta^{h}=\lim _{\varepsilon \rightarrow 0} \int_{T^{\varepsilon}} f \eta_{g}^{h}(\cdot, z) \wedge \beta^{h} .
$$

The rest of the proof is devoted to computing the limit

$$
\lim _{\varepsilon \rightarrow 0} \int_{T^{\varepsilon}} f \eta_{g}^{h}(\cdot, z) \wedge \beta^{h}
$$

by a process of passing to a residue.

In the following calculations, $\zeta$ and differential forms in it, are restricted to the manifold $T^{\ell}$. Hence

$$
\begin{gathered}
s_{j}^{g}=\frac{1}{\varepsilon^{2}} \sum_{k=1}^{q} g_{k j} \bar{g}_{k} \quad\left(\text { here } \bar{g}_{k}=\overline{g_{k}}(\zeta) \text { and } g_{k j}=g_{k j}(\zeta, z)\right), \\
\bar{\partial} s_{j}^{g}=\frac{1}{\varepsilon^{2}} \sum_{k=1}^{q} g_{k j} \overline{\partial g_{k}}
\end{gathered}
$$

and

$$
g_{1} \overline{\partial g_{1}}+\cdots+g_{q} \overline{\partial g_{q}}=0 .
$$

(Throughout this proof $\bar{\partial}=\bar{\partial}_{\zeta}$.)

It follows from (7) and (8) that $\bar{\partial} s_{j_{1}}^{g} \wedge \cdots \wedge \bar{\partial} s_{j_{q}}^{g}=0$ for $1 \leq j_{1}<\cdots<j_{q} \leq n$ and consequently

$$
\operatorname{det}[h_{1 j}, \ldots, h_{p j}, \gamma_{j}, s_{j}^{g}, \overbrace{\bar{\partial} \gamma_{j}}^{l}, \overbrace{\bar{\partial} s_{j}^{g}}^{n-p-l-2}]_{1 \leq j \leq n}=0
$$

provided that $n-p-l-2 \geq q$, i.e., $l \leq n-p-q-2$. 
Since

$$
\eta_{g}^{h}(\zeta, z)=-c(p) \sum_{l=0}^{n-p-2} \operatorname{det}[h_{1 j}, \ldots, h_{p j}, \gamma_{j}, s_{j}^{g}, \overbrace{\bar{\partial} \gamma_{j}}^{l}, \overbrace{\bar{\partial} s_{j}^{g}}^{n-p-l-2}],
$$

it follows from (9) that

$$
\eta_{g}^{h}=\tilde{\eta}+\sum_{m=0}^{q-2} \mathrm{R}_{m}
$$

where we have set

$$
\tilde{\eta}=-c(p) \operatorname{det}[h_{1 j}, \ldots, h_{p j}, \gamma_{j}, s_{j}^{g}, \overbrace{\bar{\partial} \gamma_{j}}^{n-p-q-1}, \overbrace{\bar{\partial} s_{j}^{g}}^{q-1}]_{1 \leq j \leq n}
$$

and

$$
\mathbf{R}_{m}=-c(p) \operatorname{det}[h_{1 j}, \ldots, h_{p j}, \gamma_{j}, s_{j}^{g}, \overbrace{\bar{\partial} \gamma_{j}}^{n-p-m-2}, \overbrace{\bar{\partial} s_{j}^{g}}^{m}]
$$

for $0 \leq m \leq q-2$; if $q=1$ then the terms $\mathrm{R}_{m}$ are not present (to obtain (10) we simply let $m=: n-p-l-2$, i.e., $l=n-p-m-2)$.

In order to complete the proof of Theorem 2.1 we need the following lemmas.

Lemma 3. With differential forms restricted (in $\zeta$ ) to $T^{\varepsilon}, \tilde{\eta}$ can be factorized in the following way:

$$
\tilde{\eta}=(-1)^{q n}(-1)^{(n-p)(q-1)}(-1)^{\frac{q(q-1)}{2}} \alpha^{(h, g)} \wedge \Omega^{\prime}(g)
$$

where

$$
\Omega^{\prime}(g)=(-1)^{\frac{q(q-1)}{2}} \frac{(q-1) !}{(2 \pi i)^{q}}\left(\left|g_{1}\right|^{2}+\cdots+\left|g_{q}\right|^{2}\right)^{-q} \sum_{k=1}^{q}(-1)^{k-1} \bar{g}_{k} \overline{\partial g_{1}} \wedge \cdots(k) \cdots \wedge \overline{\partial g_{q}} .
$$

Lemma 4. For $0 \leq m \leq q-2$ we have

$$
\left|\int_{\zeta \in T^{\varepsilon}} f(\zeta) \mathrm{R}_{m}(\zeta, z) \wedge \beta^{h}(\zeta)\right| \leq C_{0} \frac{\varepsilon^{2 q-1}}{\varepsilon^{2 m+1}}=O\left(\varepsilon^{2}\right)
$$

where $C_{0}$ is a constant which depends only on $z, h, g, \gamma$ and $D$.

Assuming Lemmas 3 and 4 let us complete the proof of Theorem 2.1.

Completion of the proof of Theorem 2.1. It follows from (10) that

$$
\int_{T^{\varepsilon}} f \eta_{g}^{h} \wedge \beta^{h}=\int_{T^{\varepsilon}} f \tilde{\eta} \wedge \beta^{h}+\sum_{m=0}^{q-2} \int_{T^{\varepsilon}} f \mathrm{R}_{m} \wedge \beta^{h}
$$


From Lemmas 1 and 3 we obtain

$$
\begin{aligned}
\int_{T^{e}} f \tilde{\eta} \wedge \beta^{h}= & (-1)^{q n}(-1)^{(n-p)(q-1)}(-1)^{p q}(-1)^{\frac{q(q+1)}{2}}(-1)^{\frac{q(q-1)}{2}} \\
& \cdot \int_{T^{\varepsilon}} f \alpha^{(h, g)} \wedge \Omega^{\prime}(g) \wedge \Omega(g) \wedge \beta^{(h, g)} \\
= & \int_{T^{\varepsilon}} f \alpha^{(h, g)} \wedge \beta^{(h, g)} \wedge \Omega^{\prime}(g) \wedge \Omega(g)
\end{aligned}
$$

But

$$
\begin{aligned}
\int_{T^{\varepsilon}} f \alpha^{(h, g)} \wedge \beta^{(h, g)} \wedge \Omega^{\prime}(g) \wedge \Omega(g) \\
\quad=\int_{\left\{\tau \in \mathbf{C}^{q}:|\tau|=\varepsilon\right\}}\left(\int_{Z(h) \cap\{g=\tau\} \cap \partial D} f \alpha^{(h, g)} \wedge \beta^{(h, g)}\right) \Omega^{\prime}(\tau) \wedge \Omega(\tau)
\end{aligned}
$$

(here $\Omega^{\prime}(\tau)=(-1)^{\frac{q(q-1)}{2}} \frac{(q-1) !}{(2 \pi i)^{q}}|\tau|^{-2 q} \sum_{k=1}^{q}(-1)^{k-1} \bar{\tau}_{k} \Lambda_{l \neq k} d \bar{\tau}_{l}$ and $\Omega(\tau)=d \tau_{1} \wedge$ $\left.\cdots \wedge d \tau_{q}\right)$.

Letting $\varepsilon \rightarrow 0$ we obtain from (13), also in view of (12) and the transversality assumptions made, that

$$
\begin{aligned}
\lim _{\varepsilon \rightarrow 0} \int_{T^{\varepsilon}} f \tilde{\eta} \wedge \beta^{h} & =\int_{Z(h) \cap\{g=0\} \cap \partial D} f \alpha^{(h, g)} \wedge \beta^{(h, g)} \\
& =\int_{Z(h, g) \cap \partial D} f \alpha^{(h, g)} \wedge \beta^{(h, g)} .
\end{aligned}
$$

In obtaining (14) we have also used the fact that

$$
\int_{\left\{\tau \in \mathbf{C}^{q}:|\tau|=\varepsilon\right\}} \Omega^{\prime}(\tau) \wedge \Omega(\tau)=1
$$

for all $\varepsilon>0$, by the Cauchy-Fantappiè formula in domains of $\mathbf{C}^{q}$.

On the other hand, by Lemma 4 ,

$$
\lim _{\varepsilon \rightarrow 0} \int_{T^{\varepsilon}} f \mathrm{R}_{m} \wedge \beta^{h}=0 \text { for } 0 \leq m \leq q-2 .
$$

It follows from (14), (15) and (11) that

$$
\lim _{\varepsilon \rightarrow 0} \int_{T^{\varepsilon}} f \eta_{g}^{h} \wedge \beta^{h}=\int_{Z(h, g) \cap \partial D} f \alpha^{(h, g)} \wedge \beta^{(h, g)}
$$

which together with (5) imply the integral formula of Theorem 2.1 and complete its proof.

Proof of Lemma 3. Let us recall that differential forms are restricted, in $\zeta$, to $T^{\varepsilon}$.

Since

$$
(q-1) ! \sum_{k=1}^{q}(-1)^{k-1} \bar{g}_{k} \overline{\partial g_{1}} \wedge \cdots(k) \cdots \wedge \overline{\partial g_{q}}=\operatorname{det}[\bar{g}_{k}, \overbrace{\overline{\partial g_{k}}}^{q-1}]_{1 \leq k \leq q}
$$


we obtain

$$
\Omega^{\prime}(g)=\frac{(-1)^{\frac{q(q-1)}{2}}}{(2 \pi i)^{q}} \cdot \frac{1}{g_{1}} \cdot \frac{1}{\varepsilon^{2 q}} \operatorname{det}\left[\begin{array}{cc}
\varepsilon^{2} & \overbrace{0}^{q-1} \\
\bar{g}_{k} & \frac{\partial g_{k}}{\partial \leq k \leq q}
\end{array}\right]_{2 \leq} .
$$

In obtaining (16) we used the fact that $\left|g_{1}\right|^{2}+\cdots+\left|g_{q}\right|^{2}=\varepsilon^{2}$; we also assumed that $g_{1}=g_{1}(\zeta) \neq 0$; there is no loss of generality in assuming this since the differential form $\alpha^{(h, g)} \wedge \Omega^{\prime}(g)$ is symmetric in $g_{1}, \ldots, g_{q}$, i.e., it remains unchanged under any permutation of $g_{1}, \ldots, g_{q}$. The way we obtained (16) is the following: $g_{1}$ multiplied the first row of the determinant and then we added to the first row the $k$ th-rows multiplied by $g_{k}$ (for $\left.k=2, \ldots, q\right)$. Now (16) gives

$$
\begin{aligned}
\Omega^{\prime}(g) & =(-1)^{\frac{q(q-1)}{2}} \frac{1}{(2 \pi i)^{q}} \frac{1}{g_{1}} \frac{1}{\varepsilon^{2 q-2}} \operatorname{det}\left[\overline{\partial g}_{k}\right]_{2 \leq k \leq q} \\
& =(-1)^{\frac{q(q-1)}{2}} \frac{(q-1) !}{(2 \pi i)^{q}} \frac{1}{g_{1}} \frac{1}{\varepsilon^{2 q-2}} \overline{\partial g_{2}} \wedge \cdots \wedge \overline{\partial g_{q}} .
\end{aligned}
$$

But (7) and (8) give

$$
\begin{aligned}
\bar{\partial} s_{j}^{g} & =\frac{1}{\varepsilon^{2}}\left[g_{1 j} \overline{\partial g_{1}}+\sum_{k=2}^{q} g_{k j} \overline{\partial g_{k}}\right] \\
& =\frac{1}{\varepsilon^{2}}\left[g_{1 j}\left(-\frac{1}{g_{1}} \sum_{k=2}^{q} g_{k} \overline{\partial g_{k}}\right)+\sum_{k=2}^{q} g_{k j} \overline{\partial g_{k}}\right] \\
& =\frac{1}{\varepsilon^{2}} \frac{1}{g_{1}} \sum_{k=2}^{q}\left(g_{1} g_{k j}-g_{k} g_{1 j}\right) \overline{\partial g_{k}} .
\end{aligned}
$$

Substituting (18) into $\tilde{\eta}$ we obtain

$$
\tilde{\eta}=\frac{-c(p)}{\varepsilon^{2 q} g_{1}^{q-1}} \operatorname{det}\left[h_{1 j}, \ldots, h_{p j}, \gamma_{j}, \sum_{k=1}^{q} \bar{g}_{k} g_{k j}\right.
$$

$$
\overbrace{\bar{\partial} \gamma_{j}}^{n-p-q-1}, \overbrace{\sum_{k=2}^{q}\left(g_{1} g_{k j}-g_{k} g_{1 j}\right) \overline{\partial g_{k}}}^{q-1}]
$$

or, after a computation,

(19)

$$
\begin{aligned}
\tilde{\eta}= & \frac{-c(p)(q-1) !}{\varepsilon^{2 q} g_{1}^{q-1}} \operatorname{det}[h_{1 j}, \ldots, h_{p j}, \gamma_{j}, \sum_{k=1}^{q} \bar{g}_{k} g_{k j}, \overbrace{\bar{\partial} \gamma_{j}}^{n-p-q-1}, \overbrace{g_{1} g_{k j}-g_{k} g_{1 j}}^{2 \leq k \leq q}] \\
& \wedge \overline{\partial g_{2}} \wedge \cdots \wedge \overline{\partial g_{q}} .
\end{aligned}
$$


In the determinant of (19) $j$ runs from $j=1$ to $j=n$ forming the $n$-rows of it; its last $(q-1)$-columns are indexed by $k$ which varies from $k=2$ to $k=q$ as indicated.

Using the multilinearity of the determinant in (19) in the column $\sum_{k=1}^{q} \bar{g}_{k} g_{k j}$ and in the columns indexed by $k(2 \leq k \leq q)$, we obtain (20)

$$
\begin{aligned}
\tilde{\eta}= & \frac{-c(p)(q-1) ! g_{1}^{q-2}\left(\left|g_{1}\right|^{2}+\cdots+\left|g_{q}\right|^{2}\right)}{\varepsilon^{2 q} g_{1}^{q-1}} \\
& \cdot \operatorname{det}[h_{1 j}, \ldots, h_{p j}, \gamma_{j}, g_{1 j}, \overbrace{\bar{\partial} \gamma_{j}}^{n-p-q-1}, g_{2 j}, \ldots, g_{q j}] \wedge \overline{\partial g_{2}} \wedge \cdots \wedge \overline{\partial g_{q}} .
\end{aligned}
$$

Recall that

$$
\alpha^{(h, g)}=c(p+q) \operatorname{det}[h_{1 j}, \ldots, h_{p j}, g_{1 j}, \ldots, g_{q j}, \gamma_{j}, \overbrace{\bar{\partial} \gamma_{j}}^{n-p-q-1}]
$$

where

$$
c(p+q)=(-1)^{(p+q) n}(-1)^{\frac{n(n-1)}{2}}(2 \pi i)^{p+q-n} .
$$

Now the formula of Lemma 3 follows from (21), (20) and (17). This completes the proof of Lemma 3.

Proof of Lemma 4. We have

$$
\mathbf{R}_{m}=-\frac{c(p)}{\varepsilon^{2 m+2}} \operatorname{det}[h_{1 j}, \ldots, h_{p j}, \gamma_{j}, \tilde{s}_{j}, \overbrace{\bar{\partial} \gamma_{j}}^{n-p-m-2}, \overbrace{\bar{\partial} \tilde{s}_{j}}^{m}]
$$

where $\tilde{s}_{j}=\sum_{k=1}^{q} \bar{g}_{k} g_{k j}$.

Let $A$ be so that

$$
\begin{aligned}
A \geq \max \left\{\left|\gamma_{j}(\zeta, z)\right|,\left|h_{i j}(\zeta, z)\right|,\left|g_{k j}(\zeta, z)\right|,\right. \\
\left.\left|\bar{\partial} \gamma_{j}(\zeta, z)\right|,\left|\overline{\partial h_{i}}\right|,\left|\overline{\partial g_{k}}\right|,|f(\zeta)| \text { for } \zeta \in \partial D\right\} ;
\end{aligned}
$$

$A$ depends on $z, h, g, \gamma$ and $D$; then it follows from (22) that there exists a constant $C_{1}(n, A)$, depending only on $n$ and $A$, so that

$$
\left|f(\zeta) \mathbf{R}_{m}(\zeta, z) \wedge \beta^{h}(\zeta)\right| \leq C_{1}(n, A) \frac{\varepsilon}{\varepsilon^{2 m+2}}=C_{1}(n, A) \frac{1}{\varepsilon^{2 m+1}} .
$$

It follows that

$$
\left|\int_{\zeta \in T^{\varepsilon}} f(\zeta) \mathrm{R}_{m}(\zeta, z) \wedge \beta^{h}(\zeta)\right| \leq C_{1}(n, A) \cdot \frac{1}{\varepsilon^{2 m+1}} \cdot \operatorname{vol}\left(T^{\varepsilon}\right)
$$

(here vol $\equiv(2 n-2 p-2)$-dimensional measure). 
Since

$$
\begin{aligned}
T^{\varepsilon} & =\left\{\zeta \in Z(h) \cap \partial D:\left|g_{1}(\zeta)\right|^{2}+\cdots+\left|g_{q}(\zeta)\right|^{2}=\varepsilon^{2}\right\} \\
& =\bigcup_{\left\{\tau \in \mathbf{C}^{q}:|\tau|=\varepsilon\right\}}(\{\zeta \in Z(h) \cap \partial D: g(\zeta)=\tau\})
\end{aligned}
$$

and the $(2 n-2 p-2 q-1)$-dimensional measure of each fiber $\{\zeta \in Z(h) \cap$ $\partial D: g(\zeta)=\tau\}$ is bounded, uniformly in $\tau$ (by the imposed transversality conditions), it follows that

$$
\operatorname{vol}\left(T^{\varepsilon}\right) \leq C_{2} \varepsilon^{2 q-1}
$$

for some constant $C_{2}$ depending only on $h, g$ and $D$.

Now (23) and (24) imply the estimate of Lemma 4 and complete its proof.

The following theorem is a consequence of Theorem 2.1. It gives a relation among certain integrals associated to CR-functions defined on parts of the boundaries of two varieties whose intersection is at least 1-dimensional. The setting of this theorem is similar to the one of Theorem 2.1 but with some additional transversality assumptions which we describe next. Let us consider the setting of Theorem 2.1 with the additional assumptions that $|\nabla g| \neq 0$ on $Z(g) \cap \partial D$ and that $Z(g)$ meets $\partial D$ transversally. Furthermore let us consider $\Gamma, \widetilde{\Gamma} \subset Z(h) \cap \partial D$ open subsets of $Z(h) \cap \partial D$ and $\Delta, \widetilde{\Delta} \subset Z(g) \cap \partial D$ open subsets of $Z(g) \cap \partial D$ so that $Z(h, g) \cap \partial D \subset \Gamma \cap \Delta, \bar{\Gamma} \subset \widetilde{\Gamma}, \bar{\Delta} \subset \widetilde{\Delta}$ and $\partial \Gamma$ and $\partial \Delta$ smooth. In this setting we will prove the following theorem.

Theorem 2.2. For a smooth $C R$-function $f$ on $\widetilde{\Gamma} \cup \widetilde{\Delta}$ and for $z \in Z(h, g) \cap D$ the following formula holds:

$$
\begin{aligned}
\int_{\Gamma} f \alpha^{h}(\cdot, z) \wedge \beta^{h}-\int_{\partial \Gamma} f \eta_{g}^{h}(\cdot, z) \wedge \beta^{h} \\
=\int_{\Delta} f \alpha^{g}(\cdot, z) \wedge \beta^{g}-\int_{\partial \Delta} f \eta_{h}^{g}(\cdot, z) \wedge \beta^{g} .
\end{aligned}
$$

Proof. First let us point out that $\eta_{h}^{g}(\cdot, z)$ is a $\bar{\partial}_{\zeta}$-primitive of $\alpha^{g}(\cdot, z)$ on the manifold $(Z(g)-Z(g, h)) \cap \operatorname{Dom}(\gamma(\cdot, z))$. It follows from Theorem 2.1 that

$$
\int_{\Gamma} f \alpha^{h}(\cdot, z) \wedge \beta^{h}-\int_{\partial \Gamma} f \eta_{g}^{h}(\cdot, z) \wedge \beta^{h}=\int_{Z(h, g) \cap \partial D} f \alpha^{(h, g)} \wedge \beta^{(h, g)}
$$

and

$$
\int_{\Delta} f \alpha^{g}(\cdot, z) \wedge \beta^{g}-\int_{\partial \Delta} f \eta_{h}^{g}(\cdot, z) \wedge \beta^{g}=\int_{Z(g, h) \cap \partial D} f \alpha^{(g, h)} \wedge \beta^{(g, h)} .
$$

Since $\alpha^{(h, g)} \wedge \beta^{(h, g)}=\alpha^{(g, h)} \wedge \beta^{(g, h)},(1)$ and (2) imply the formula of Theorem 2.2 and complete its proof.

Remark. A particular case of Theorem 2.2 is when $\Gamma=Z(h) \cap \partial D$ and $\Delta=$ $Z(g) \cap \partial D$ in which case the formula of the theorem becomes

$$
\int_{Z(h) \cap \partial D} f \alpha^{h}(\cdot, z) \wedge \beta^{h}=\int_{Z(g) \cap \partial D} f \alpha^{g}(\cdot, z) \wedge \beta^{g}
$$


for $f$ a CR-function on $(Z(h) \cup Z(g)) \cap \partial D$ and $z \in Z(h, g) \cap D$. Recall that it is part of the assumptions, under which (I) was proved, that $p+q \leq n-1$. Another assumption that we used in the proof of $(\mathrm{I})$ is the following:

$$
\begin{aligned}
& |\nabla(h, g)| \neq 0 \text { on } Z(h, g) \cap \partial D \text { and } Z(h, g) \\
& \text { meets } Z(h) \cap \partial D \text { and } Z(g) \cap \partial D \text { transversally. }
\end{aligned}
$$

Obviously (II) is not needed in the statement of the integral formula (I), i.e., the integrals in (I) make sense without the assumption (II); however we do not know how to prove (I) without (II). Recall that the way (I) is proved is by comparing each side of it to the integral $\int_{Z(h, g) \cap \partial D} f \alpha^{(h, g)} \wedge \beta^{(h, g)}$ which becomes improper if $|\nabla(h, g)|=0$ at some point of $Z(h, g) \cap \partial D$ and the contour $Z(h, g) \cap \partial D$ is not a smooth manifold if $Z(h, g)$ fails to meet $Z(h) \cap$ $\partial D$ transversally.

There is an interesting case in which (I) holds without the assumption (II): if $f$ extends as a holomorphic function in a neighborhood (in $\left.\mathbf{C}^{n}\right)$ of $(Z(h) \cup$ $Z(g)) \cap \partial D$ then a deformation argument based on Sard's theorem proves (I) without (II) (by reducing the general case to the case in which (II) holds). One more case in which we know that (I) holds without (II) (without even the condition: $p+q \leq n-1)$ is the following: If $f$ extends to a CR-function on $\partial D$ then (by Theorem 2.1) each side of $(\mathrm{I})$ is equal to $\int_{\partial D} f \omega^{\prime}(\gamma)(\cdot, z) \wedge \omega$ where $\omega^{\prime}(\gamma)(\cdot, z) \wedge \omega$ is the Cauchy-Fantappiè kernel associated to $\gamma$ (see $\S 3$ for this notation). Of course $z$ is assumed to be in $Z(h) \cap Z(g) \cap D \quad(Z(h) \cap Z(g) \cap D$ may be just a finite set of points). In conclusion, let us point out that what we said, in this remark, about (I) holds also for the more general formula of Theorem 2.2.

\section{A CRITERION FOR EXTENDABILITY OF CR-FUNCTIONS}

In this section we specialize in the case of domains in $\mathbf{C}^{n}$ and the $\bar{\partial}$ primitive of the Cauchy-Fantappiè kernel given by Theorem 1.1. We will use these primitives to state and prove a criterion for holomorphic extendability of CR-functions which are defined in certain parts of the boundary of a domain in $\mathbf{C}^{n}$.

Let $D, G$ and $\gamma$ be as in $\S 1$ with $G$ being pseudoconvex. Recall that the Cauchy-Fantappiè kernel associated to $\gamma$ is the $(n, n-1)$-form $\omega^{\prime}(\gamma)(\zeta, z) \wedge$ $\omega(\zeta)$ where

$$
\omega^{\prime}(\gamma)=c(0)(n-1) ! \sum_{j=1}^{n}(-1)^{j-1} \gamma_{j} \bigwedge_{k \neq j} \bar{\partial}_{\zeta} \gamma_{k}, \quad \omega(\zeta)=d \zeta_{1} \wedge \cdots \wedge d \zeta_{n}
$$

and

$$
c(0)=(-1)^{\frac{n(n-1)}{2}}(2 \pi i)^{-n} .
$$

Let $h: G \rightarrow \mathbf{C}^{p}$ be a holomorphic map with $p \leq n-1$. Since we assume $G$ to be pseudoconvex, there exists a Hefer decomposition $\left\{h_{i j}\right\}$ of $h$. It follows 
from Theorem 1.1 that if

$$
\eta_{h}^{0}(\zeta, z)=-c(0) \sum_{l=0}^{n-2} \operatorname{det}[\gamma_{j}, s_{j}^{h}, \overbrace{\bar{\partial} \gamma_{j}}^{l}, \overbrace{\bar{\partial} s_{j}^{h}}^{n-l-2}]_{1 \leq j \leq n}
$$

with

$$
s_{j}^{h}(\zeta, z)=\left(\sum_{i=1}^{p}\left|h_{i}(\zeta)\right|^{2}\right)^{-1} \sum_{i=1}^{p} h_{i j}(\zeta, z) \overline{h_{i}(\zeta)}
$$

then

$$
\bar{\partial}_{\zeta} \eta_{h}^{0}(\zeta, z)=\omega^{\prime}(\gamma)(\zeta, z)
$$

and

$$
\bar{\partial}_{\zeta}\left[\eta_{h}^{0}(\zeta, z) \wedge \omega(\zeta)\right]=\omega^{\prime}(\gamma)(\zeta, z) \wedge \omega(\zeta)
$$

for a fixed $z \in Z(h) \cap D$ and $\zeta^{\prime} \in \operatorname{Dom}(\gamma(\cdot, z))-Z(h)$. (Throughout this section $\bar{\partial}=\bar{\partial}_{\zeta}$.)

Next we compare certain integrals of such primitives corresponding to two holomorphic maps $h$ and $g$. To make it precise let $g: G \rightarrow \mathbf{C}^{q}$ be another holomorphic map and $\left\{g_{k j}\right\}$ be a Hefer decomposition of $g$; then we can associate to $g$ and $\left\{g_{k j}\right\}$ the differential form $\eta_{g}^{0}(\cdot, z)$ which is a $\bar{\partial}_{\zeta}$-primitive of $\omega^{\prime}(\gamma)(\cdot, z)$ on $\operatorname{Dom}(\gamma(\cdot, z))-Z(g)$. Let $\Gamma, \tilde{\Gamma} \subset \partial D$ be open subsets of $\partial D$ so that $\bar{\Gamma} \subset \widetilde{\Gamma}$ and $\partial \Gamma$ is smooth. Suppose that $(Z(h) \cup Z(g)) \cap \partial D \subset \Gamma$. If $p+q \leq n-1$ and the varieties $Z(h)$ and $Z(g)$ satisfy the geometric assumptions of Theorem 2.2 then the following proposition holds:

Proposition 3.1. For a smooth CR-function $f$ on $\widetilde{\Gamma}$ and a fixed $z \in Z(h) \cap$ $Z(g) \cap D$ we have

$$
\int_{\zeta \in \partial \Gamma} f(\zeta) \eta_{h}^{0}(\zeta, z) \wedge \omega(\zeta)=\int_{\zeta \in \partial \Gamma} f(\zeta) \eta_{g}^{0}(\zeta, z) \wedge \omega(\zeta)
$$

Proof. It follows from Theorem 2.1 that

$$
\int_{\Gamma} f \omega^{\prime}(\gamma)(\cdot, z) \wedge \omega-\int_{\partial \Gamma} f \eta_{h}^{0}(\cdot, z) \wedge \omega=\int_{Z(h) \cap \partial D} f \alpha^{h}(\cdot, z) \wedge \beta^{h}
$$

and

$$
\int_{\Gamma} f \omega^{\prime}(\gamma)(\cdot, z) \wedge \omega-\int_{\partial \Gamma} f \eta_{g}^{0}(\cdot, z) \wedge \omega=\int_{Z(g) \cap \partial D} f \alpha^{g}(\cdot, z) \wedge \beta^{g}
$$

Now (1) follows from (2), (3) and Theorem 2.2 (see the remark following the proof of Theorem 2.2).

Remarks. (a) The various smoothness and transversality conditions that we used to prove (1) (these are the conditions assumed in Theorem 2.2) are not needed in the statement of the integral formula (1); we do not know whether (1) holds without these conditions except in two cases which we describe next. 
(b) If $p=1$ (i.e., $h=h_{1}$ ) then $s_{j}^{h}$ reduces to $s_{j}^{h}=(h(\zeta))^{-1} h_{1 j}(\zeta, z)$ and, since $\bar{\partial}_{\zeta} s_{j}^{h}=0$, most of the terms in the sum defining $\eta_{h}^{0}$ vanish and we obtain, in this case:

$$
\eta_{h}^{0}=-\frac{c(0)}{h(\zeta)} \operatorname{det}[\gamma_{j}, h_{1 j}, \overbrace{\bar{\partial} \gamma_{j}}^{n-2}] .
$$

The case $\gamma_{j}=|\zeta-z|^{-2}\left(\bar{\zeta}_{j}-\bar{z}_{j}\right)$ of it is considered in [3, 5 and 7]. If $p=q=1$ (i.e., $h=h_{1}$ and $g=g_{1}$ ) then

$$
\eta_{h}^{0}-\eta_{g}^{0}=\bar{\partial}\left(-c(0)(h(\zeta) g(\zeta))^{-1} \operatorname{det}[\gamma_{j}, h_{1 j}, g_{1 j}, \overbrace{\bar{\partial} \gamma_{j}}^{n-3}]\right)
$$

for a fixed $z \in Z(h) \cap Z(g)$ and $\zeta \in \operatorname{Dom}(\gamma(\cdot, z))-(Z(h) \cup Z(g))$; this is proved in [3] for $\gamma_{j}=|\zeta-z|^{-2}\left(\bar{\zeta}_{j}-\bar{z}_{j}\right)$; the proof for arbitrary $\gamma$ is similar.

Thus, in the case $p=q=1(n \geq 3)$, (1) follows immediately from (4) without any geometric restrictions on $Z(h)$ or $Z(g)$.

(c) If $f$ extends to a neighborhood (in $\bar{D})$ of $(Z(h) \cup Z(g)) \cap \partial D$ as a holomorphic function then a deformation argument based on Sard's theorem proves that (1) holds without the geometric restrictions on $Z(h)$ and $Z(g)$. Notice also that $f$ extends to a holomorphic function in a neighborhood (in $\bar{D}$ ) of $(Z(h) \cup Z(g)) \cap \partial D$ if, for example, at each point of $(Z(h) \cup Z(g)) \cap \partial D$, the Levi form of $\partial D$ has at least one positive eigenvalue.

Next we specialize further in the case $\gamma_{j}=b_{j}=:|\zeta-z|^{-2}\left(\bar{\zeta}_{j}-\bar{z}_{j}\right)$ in which case the Cauchy-Fantappiè kernel $\omega^{\prime}(\gamma) \wedge \omega$ is the Bochner-Martinelli kernel $\omega^{\prime}(b) \wedge \omega$. We will denote by $\mu_{h}$ the $\bar{\partial}$-primitive $\eta_{h}^{0}$ of $\omega^{\prime}(b) \wedge \omega$ on $G-Z(h)$, i.e.,

$$
\mu_{h}=-c(0) \sum_{l=0}^{n-2} \operatorname{det}[b_{j}, s_{j}^{h}, \overbrace{\bar{\partial} b_{j}}^{l}, \overbrace{\bar{\partial} s_{j}^{h}}^{n-l-2}] .
$$

Now we will restrict attention to the case $p \leq n-2$. If we fix $\Gamma, \widetilde{\Gamma} \subset \partial D$ with $\bar{\Gamma} \subset \widetilde{\Gamma}, \partial \Gamma$ smooth and $Z(h) \cap \partial D \subset \Gamma$ and $z \in Z(h)$ then the integral

$$
\int_{\zeta \in \partial \Gamma} f(\zeta) \mu_{h}(\zeta, z) \wedge \omega(\zeta)
$$

is independent of the chosen Hefer decomposition $\left\{h_{i j}\right\}$ of $h$. Indeed, if $\left\{h_{i j}^{\prime}\right\}$ is another Hefer decomposition of $h$ and $\mu_{h}^{\prime}$ is the corresponding $\bar{\partial}$-primitive of $\omega^{\prime}(b) \wedge \omega$ on $G-Z(h)$ then, since $G-Z(h)$ is $(n-2)$-complete (since $p \leq n-2)$ the difference $\mu_{h}-\mu_{h}^{\prime}$ is $\bar{\partial}$-exact on $G-Z(h)$ and consequently, by Stokes' theorem and the fact that $f$ is a CR-function:

$$
\int_{\partial \Gamma} f \mu_{h}(\cdot, z) \wedge \omega=\int_{\partial \Gamma} f \mu_{h}^{\prime}(\cdot, z) \wedge \omega
$$


Also notice that if

$$
\int_{\partial \Gamma} f \mu_{h}(\cdot, z) \wedge \omega=\int_{\partial \Gamma} f \mu_{g}(\cdot, z) \wedge \omega
$$

holds for one $\Gamma$ then it holds for any other $\Gamma$; to see this let $\Gamma^{\prime} \subset \widetilde{\Gamma}$ be another one with $\partial \Gamma^{\prime}$ smooth and $Z(h) \cap \partial D \subset \Gamma^{\prime}$; then

$$
\int_{\partial \Gamma^{\prime}} f \mu_{h}(\cdot, z) \wedge \omega-\int_{\partial \Gamma} f \mu_{h}(\cdot, z) \wedge \omega=\int_{\left(\Gamma^{\prime}-\Gamma\right) \cup\left(\Gamma-\Gamma^{\prime}\right)} f \omega^{\prime}(b) \wedge \omega
$$

with the various parts of $\left(\Gamma^{\prime}-\Gamma\right) \cup\left(\Gamma-\Gamma^{\prime}\right)$ appropriately oriented; since the right-hand side of (6) is independent of $h$, it follows that (5) implies

$$
\int_{\partial \Gamma^{\prime}} f \mu_{h}(\cdot, z) \wedge \omega=\int_{\partial \Gamma^{\prime}} f \mu_{g}(\cdot, z) \wedge \omega
$$

provided that $Z(g) \cap \partial D \subset \Gamma^{\prime}$.

With these comments in mind we will prove a criterion for holomorphic extendability of CR-functions defined on certain subsets of $\partial D$. First we describe the setting.

Let $E \subset \partial D$ be a closed subset of $\partial D$ so that each connected component of $\partial D-E$ contains a peak point for $\mathscr{O}(\bar{D})$. For each $z \in G-\partial D$ let

$$
\mathscr{P}_{z}=\left\{h: G \rightarrow \mathbf{C}^{n-2}: h \text { holomorphic, } z \in Z(h) \text { and } Z(h) \cap E=\varnothing\right\} .
$$

With this notation we will prove the following theorem.

Theorem 3.1. Suppose that $\mathscr{P}_{z} \neq \varnothing$ for each $z \in G-\partial D$. Let $f$ be a smooth $C R$-function on $\partial D-E$. Then a necessary and sufficient condition that $f$ extends to a holomorphic function in $D$ is

$$
\int_{\zeta \in \partial \Gamma} f(\zeta) \mu_{h}(\zeta, z) \wedge \omega(\zeta)=\int_{\zeta \in \partial \Gamma} f(\zeta) \mu_{g}(\zeta, z) \wedge \omega(\zeta)
$$

for $h, g \in \mathscr{P}_{z}, \Gamma \supset(Z(h) \cup Z(g)) \cap \partial D$ open (in $\partial D$ ) with $\bar{\Gamma} \subset \partial D-E$ and $\partial \Gamma$ smooth and $z \in G-\partial D$.

Proof. (M) is necessary: In this direction we assume $f$ extends to a holomorphic function in $D$, denoted by $f$ too. Then it follows from the BochnerMartinelli formula and the fact that $d\left[f \mu_{h}(\cdot, z) \wedge \omega\right]=\omega^{\prime}(b) \wedge \omega$ that

$$
f(z)=\int_{\Gamma} f \omega^{\prime}(b)(\cdot, z) \wedge \omega-\int_{\partial \Gamma} f \mu_{h}(\cdot, z) \wedge \omega
$$

(see also [3, p. 185]).

Similarly,

$$
f(z)=\int_{\Gamma} f \omega^{\prime}(b)(\cdot, z) \wedge \omega-\int_{\partial \Gamma} f \mu_{g}(\cdot, z) \wedge \omega .
$$

Now (M) follows from (1) and (2); this proves that (M) is necessary.

(M) is sufficient: In this direction we assume $(\mathbf{M})$ holds and we will prove that $f$ extends to a holomorphic function in $D$. The way we define such an 
extension is the following: for $z \in G-\partial D$, choose an $h \in \mathscr{P}_{z}$ and a $\Gamma$ with $\Gamma \supset Z(h) \cap \partial D, \partial \Gamma$ smooth and $\bar{\Gamma} \subset \partial D-E$; define $F(z)$ by

$$
F(z)=\int_{\zeta \in \Gamma} f(\zeta) \omega^{\prime}(b)(\zeta, z) \wedge \omega(\zeta)-\int_{\zeta \in \partial \Gamma} f(\zeta) \mu_{h}(\zeta, z) \wedge \omega(\zeta)
$$

Now condition (M) guarantees that $F(z)$ is well defined, i.e., it is independent of the choice of $h$ (and its Hefer decomposition) and of the choice of $\Gamma$.

It remains to prove that $\left.F\right|_{D}(F$ restricted to $D)$ is a holomorphic extension of $f$.

First let us prove that $F$ is holomorphic in $G-\partial D$. For this purpose fix a $z^{0} \in G-\partial D$ and choose an $h^{0} \in \mathscr{P}_{z^{0}}$; of course, $h^{0}$ depends on $z^{0}$ but if $z$ is sufficiently close to $z^{0}$ then $h^{0}-h^{0}(z) \in \mathscr{P}_{z}$, so that we may use the kernel $\mu_{h^{0}-h^{0}(z)}$ in the integral defining $F(z)$, i.e.,

$$
F(z)=\int_{\Gamma} f \omega^{\prime}(b)(\cdot, z) \wedge \omega-\int_{\partial \Gamma} f \mu_{h^{0}-h^{0}(z)}(\cdot, z) \wedge \omega ;
$$

consequently, for $z$ close to $z^{0}$,

$$
\begin{aligned}
\frac{\partial F}{\partial \bar{z}_{k}}= & \int_{\zeta \in \Gamma} f(\zeta) \frac{\partial \omega^{\prime}(b)}{\partial \bar{z}_{k}}(\zeta, z) \wedge \omega(\zeta) \\
& -\int_{\zeta \in \partial \Gamma} f(\zeta) \frac{\partial \mu_{h^{0}-h^{0}(z)}}{\partial \bar{z}_{k}}(\zeta, z) \wedge \omega(\zeta) .
\end{aligned}
$$

Let

where

$$
\varphi_{k}(\zeta, z)=-\frac{c(0)}{\zeta_{k}-z_{k}} \operatorname{det}[b_{j}, \delta_{k j}, \overbrace{\bar{\partial} b_{j}}^{n-2}]_{1 \leq j \leq n}
$$

$$
\delta_{k j}= \begin{cases}1 & \text { if } k=j \\ 0 & \text { if } k \neq j\end{cases}
$$

$\varphi_{k}$ is simply a $\bar{\partial}$-primitive of $\omega^{\prime}(b) \wedge \omega$ on $\mathbf{C}^{n}-\left\{\zeta \in \mathbf{C}^{n}: \zeta_{k}=z_{k}\right\}$ (for a fixed $k, 1 \leq k \leq n)$.

A computation shows that

$$
\frac{\partial \varphi_{k}}{\partial \bar{z}_{k}}=(n-1) \frac{\zeta_{k}-z_{k}}{|\zeta-z|^{2}} \varphi_{k}(\zeta, z)
$$

(see $[3$, p. 180]).

It follows from (4) that $\partial \varphi_{k}(\cdot, z) / \partial \bar{z}_{k}$ is extended from $\mathbf{C}^{n}-\left\{\zeta \in \mathbf{C}^{n}: \zeta_{k}=\right.$ $\left.z_{k}\right\}$ to $\mathbf{C}^{n}-\{z\}$ as a smooth form and that

$$
\bar{\partial}_{\zeta}\left[\frac{\partial \varphi_{k}}{\partial \bar{z}_{k}}\right]=\frac{\partial \omega^{\prime}(b)}{\partial \bar{z}_{k}} \text { in } \mathbf{C}^{n}-\{z\}
$$

On the other hand, since the set $G-Z\left(h^{0}-h^{0}(z)\right)$ is $(n-2)$-complete and $\bar{\partial}_{\zeta}\left[\frac{\partial \varphi_{k}}{\partial \bar{z}_{k}}-\frac{\partial \mu_{h^{0}-h^{0}(z)}}{\partial \bar{z}_{k}}\right]=\frac{\partial \omega^{\prime}(b)}{\partial \bar{z}_{k}}-\frac{\partial \omega^{\prime}(b)}{\partial \bar{z}_{k}}=0 \quad$ in $G-Z\left(h^{0}-h^{0}(z)\right)$, 
it follows that

$$
\frac{\partial \varphi_{k}}{\partial \bar{z}_{k}}-\frac{\partial \mu_{h^{0}-h^{0}(z)}}{\partial \bar{z}_{k}}=\bar{\partial}_{\zeta} \nu(\cdot, z)
$$

for some $(0, n-3)$-form $\nu(\zeta, z)$. Hence

$$
\begin{aligned}
\int_{\partial \Gamma} f \frac{\partial \varphi_{k}}{\partial \bar{z}_{k}} \wedge \omega & -\int_{\partial \Gamma} f \frac{\partial \mu_{h^{0}-h^{0}(z)}}{\partial \bar{z}_{k}} \wedge \omega=\int_{\partial \Gamma} f \bar{\partial} \nu(\cdot, z) \wedge \omega \\
= & \int_{\partial \Gamma} d[f \nu(\cdot, z) \wedge \omega]=0 .
\end{aligned}
$$

Thus (3), in view of (6), becomes

$$
\begin{aligned}
\frac{\partial F}{\partial \bar{z}_{k}}(z) & =\int_{\Gamma} f \frac{\partial \omega^{\prime}(b)}{\partial \bar{z}_{k}} \wedge \omega-\int_{\partial \Gamma} f \frac{\partial \varphi_{k}}{\partial \bar{z}_{k}} \wedge \omega \\
& =\int_{\Gamma} f \frac{\partial \omega^{\prime}(b)}{\partial \bar{z}_{k}} \wedge \omega-\int_{\Gamma} d\left[f \frac{\partial \varphi_{k}}{\partial \bar{z}_{k}} \wedge \omega\right] \\
& =\int_{\Gamma} f \frac{\partial \omega^{\prime}(b)}{\partial \bar{z}_{k}} \wedge \omega-\int_{\Gamma} f \frac{\partial \omega^{\prime}(b)}{\partial \bar{z}_{k}} \wedge \omega=0
\end{aligned}
$$

The third equation in (7) follows from (5). This proves that $F$ is holomorphic in $G-\partial D$. That $\left.F\right|_{D}$ has boundary values the function $f$ on $\partial D-E$ is proved exactly as in [3, pp. 188-189]; see also [4, p. 121]. This completes the proof of Theorem 3.1.

\section{REFERENCES}

1. I. Aizenberg and A. Yuzhakov, Integral representations and residues in multidimensional complex analysis, Transl. Math. Monos., vol. 58, Amer. Math. Soc., Providence, R.I., 1983.

2. T. Hatziafratis, Integral representation formulas on analytic varieties, Pacific J. Math. 123 (1986), 71-91.

3. G. Lupacciolu, A theorem on holomorphic extension of CR-functions, Pacific J. Math. 124 (1986), 177-191.

4. _ _ Holomorphic continuation in several complex variables, Pacific J. Math. 128 (1987), 117-126.

5. G. Lupacciolu and G. Tomassini, Un teorema di estensione per le CR-funzioni, Ann. Mat. Pura Appl. 137 (1984), 257-263.

6. E. L. Stout, An integral formula for holomorphic functions on strictly pseudoconvex hypersurfaces, Duke Math. J. 42 (1975), 347-356.

7. _ Analytic continuation and boundary continuity of functions of several complex variables, Proc. Roy. Soc. Edinburgh 89A (1981), 63-74.

Department of Mathematics, University of Washington, Seattle, Washington 98195 